of the historical development of the theory. Endless excursions into topics in the history of statistics and probability (which have been better described by other recent writers) obscure the main theme, while the long quotations and cramped footnotes in a minuscule type further detract from a coherent account.

The author seems to have become fascinated by the Galton and Pearson archives in University College London, resulting in an overload of peripheral material. Thus the large number of diagrams oddly includes both Alfred Russel Wallace's sketch of an irregular frequency curve, and the distribution of head breadth in W F. R. Weldon's crabs, while a whole page is devoted to "Bowditch's composite photograph of 287 American female college students" (all these from the Galton and Pearson archives). But the series of sunspot numbers is nowhere to be found, either tabulated or depicted, and poor Schwabe does not even get a mention.

The history of probability and statistics has been well-served by recent writers, but there is still a gap to be filled around the turn of the century. We lack a biography of Karl Pearson, while W. Stanley Jevons, F. Y. Edgeworth and Yule are only now beginning to emerge from the shadows. Statistical Visions in Time contains much relevant material for this period, but not in a coherent form. Indeed, the book is rather like a time series itself: the underlying message is difficult to interpret because of the overlay of random noise. A shorter series with less noise would have been more informative.

A. W. F. Edwards is at Gonville and Caius College, University of Cambridge, Cambridge CB2 1TA, UK.

\section{Life is like a game of chess}

\section{Emergence: From Chaos to Order}

by John Holland

Addison-Wesley: 1998. 258pp. \$25

\section{Hanspeter Mallot}

Nature is full of wonders. Trees and flowers grow from inconspicuous seeds, simple physical laws underlie complex phenomena such as climate change or the movement of celestial bodies, and even man-made games such as chess or Go keep generating unsolved problems. Do such phenomena form a welldefined class or is it only the amazement they generate in the observer that groups them together? John Holland takes the view that there is a common principle or rule underlying phenomena where "much comes from little"; he adopts the term "emergence" to characterize this principle.

In his latest book, Holland examines a number of arenas of emergent behaviour,

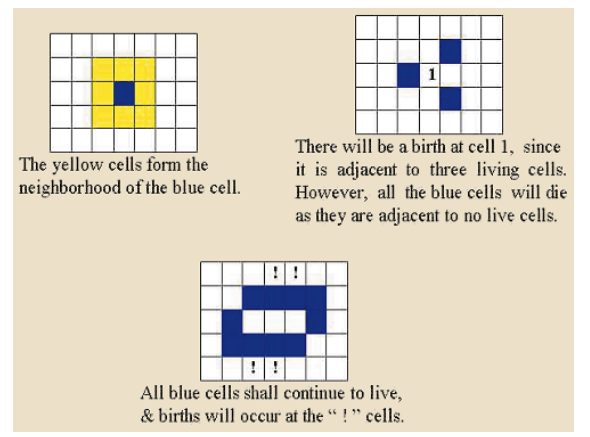

Game of Life: the rules.

including board games (chess and checkers), machine learning, cellular automata and neural networks. But what exactly is emergence? Holland is not very explicit about this question, leaving the reader with hints such as "much coming from little" or "the whole is more than the sum of its parts". In order to make that into a definition of any value, one needs to say something about the measures used to judge much and little or more and less. No such attempt is made in the book, and, in fact, I doubt that a convincing definition (except for the observer's amazement) can be given. Instead, Holland uses the term "emergence" in an associative and exploratory way.

By and large, emergence seems to be something that arises in the context of simple theories, games or mathematical axiom systems, that is, in models of the world, rather than in the world itself. In this sense, unanticipated predictions may emerge from a physical theory or certain chess strategies may emerge from the rules of the game. One could call this a logical notion of emergence since it is confined to the realm of theory. A related, epistemological notion of emergence views it as the inverse of reduction. This again is a model-immanent view that is not much different from surprise or amazement.

Holland also uses the term in other, more puzzling ways, where "emergence" seems to refer to the causes of real world phenomena. One view is psychological in nature: learning and creativity are called emergent since they lead to behavioural competences or insights that did not exist before emergence occurred. In its most equivocal version, the term occurs on the first page of the book, where we read that "we will not understand life and living organisms until we understand emergence". Here, emergence appears to be a natural phenomenon that causes the origin of life. It is clearly this connotation of a vital force that gives the term its appeal, even though Holland tries to avoid such associations in later parts of the book.

What do we gain by adopting the term "emergence" as a scientific notion? Do we really learn something about the origin of life, say, if we understand machine learning? Of course, there is always the possibility of cross-fertilizations between far distant fields of science, but this is not the kind of insight the author seems to promise. What he is interested in is a general theory of complexity of which emergence would be a part.

The book is written for a generally educated reader. The examples selected for the illustration of emergent behaviour are very interesting. (In fact, I think that this interest is what measures "much and little" in Holland's explication of emergence.) Holland uses John Conway's Game of Life, computer programs learning board games, and neural networks to illustrate what he has identified as building blocks of emergence: the interaction of simple mechanisms. While making this general point, there are a number of inaccuracies in the details that limit the value of Holland's presentation. To mention just one example, the proposed mechanism of visual pattern recognition based on Hebbian learning and saccadic eye movements is biologically highly implausible, not least for the exceedingly long time required to recognize even simple triangles.

Towards the end of the book, Holland states: "at the broadest level, this book's thesis has been that models and model building underlie much, perhaps most, human intellectual endeavor". While this statement is too broad to be challenged, it makes an important point about the book: Holland is not so much interested in the analysis of natural systems, but in the construction of artificial ones. In this context, the problem of emergence may actually be a genuine one. The empirical part of natural science is reductionist or top-down, whereas in the construction of systems (and theories) one proceeds bottom-up. So if we view emergence as the inverse of reduction, here it may have its valid place.

Hanspeter Mallot is in the Max Planck Institute for Biological Cybernetics, Spemannstr. 38, D-72076 Tübingen, Germany.

\section{correction}

In the 27 August issue (Nature 394, 844-845; 1998) we published a review of The Story of Interferon by Kari Cantell. The review repeated allegations made in the book about the late Frank Rauscher, a former senior vicepresident of research (not president, as stated) of the American Cancer Society. The allegations concern the purchase by the ACS of interferon from a US company, Life Sciences, in preference to the Finnish Red Cross Blood Transfusion Service. Nature and Dr Cantell accept that Dr Rauscher was not one of the founders of Life Sciences and that neither Dr Rauscher nor anyone else at the ACS had any financial interest in the company, or stood to gain financially from the transaction. We apologize to the ACS and to Frank Rauscher's family for this misrepresentation. 